Check for updates

Cite this: RSC Adv., 2019, 9, 5189

Received 26th November 2018 Accepted 3rd February 2019

DOI: $10.1039 / c 8 r a 09700 c$

rsc.li/rsc-advances

\section{Nitrogen and sulfur-codoped porous carbon derived from a BSA/ionic liquid polymer complex: multifunctional electrode materials for water splitting and supercapacitors $\uparrow$}

\author{
Xiaojun Liu, Junrui Yu, ${ }^{b}$ Honghong Song, ${ }^{b}$ Pengfei Song, ${ }^{b}$ Rongming Wang (DD ${ }^{b}$ \\ and Yubing Xiong (D) *ab
}

\begin{abstract}
Bovine serum albumin (BSA) was complexed with a hydrophobic ionic liquid polymer (PIL) via electrostatic interaction to fabricate a carbon precursor. Then, a novel nitrogen (N) and sulfur (S) codoped micro-/ mesoporous carbon (NSPC) was obtained via direct carbonization of the interpolyelectrolyte BSA aPIL complex. The newly developed NSPC materials exhibited excellent HER/OER electrocatalytic activity and stability, as well as outstanding capacitance performance. Remarkably, NSPC pyrolyzed at 1000 degrees (NSPC-1000) presented an overpotential as low as $172 \mathrm{mV}$ vs. RHE (without iR correction) to achieve a current density of $10 \mathrm{~mA} \mathrm{~cm}{ }^{-2}$ and a Tafel slope of $44.3 \mathrm{mV} \mathrm{dec}^{-1}$ in $0.5 \mathrm{M} \mathrm{H}_{2} \mathrm{SO}_{4}$ for $\mathrm{HER}$, as well as a low overpotential of $460 \mathrm{mV}$ vs. RHE in $0.1 \mathrm{M} \mathrm{KOH}$ for OER. Furthermore, NSPC-1000 offers a specific capacitance as high as $495 \mathrm{~F} \mathrm{~g}^{-1}$ at a current density of $0.1 \mathrm{~A} \mathrm{~g}^{-1}$. Such excellent performance of NSPC in electrocatalytic water splitting and supercapacitors originates from the synergistic effects of its N/Scodoping and micro-/mesoporous hierarchical architecture. Our facile protocol through combining biomacromolecules and synthetic polymers offers a new strategy in the development of effective, readily scalable and metal-free heteroatom-doped carbon materials for energy-related applications.
\end{abstract}

\section{Introduction}

Spurred by the urgent demand for green and renewable energy technologies, great efforts have been applied worldwide for the exploration of alternatives to traditional fossil fuels. ${ }^{\mathbf{1 , 2}}$ Electrocatalytic water splitting to $\mathrm{H}_{2}$ and $\mathrm{O}_{2}$ via the hydrogen evolution reaction (HER) and oxygen evolution reaction (OER) affords an appealing solution for addressing the issues associated with global warming and the energy crisis. ${ }^{3,4}$ Hydrogen, as an abundant and renewable clean fuel, is an ideal candidate to replace fossil fuels. Simultaneously, producing $\mathrm{O}_{2}$ from water splitting is also related to a variety of energy storage systems. ${ }^{5-7}$ As benchmark electrocatalysts, platinum is sufficiently active in the HER and ruthenium/iridium oxides are highly efficient for OER. However, both of them suffer from the weaknesses of high cost and non-renewability. ${ }^{8-12}$ Therefore, highly efficient and

\footnotetext{
${ }^{a}$ Department of Chemistry, Zhejiang Sci-Tech University, Hangzhou 310018, P. R. China.E-mail: yubing_xiong@163.com

${ }^{b}$ Key Laboratory of Eco-Environment-Related Polymer Materials, Ministry of Education, College of Chemistry and Chemical Engineering, Northwest Normal University, Lanzhou 730070, P. R. China

$\dagger$ Electronic supplementary information (ESI) available: ${ }^{1} \mathrm{H}$ and ${ }^{13} \mathrm{C}$ NMR spectra, GPC curve, EDX, element analysis, SEM and HRTEM images, photographs, electrochemical impedance spectroscopy and CV profiles. See DOI: 10.1039/c8ra09700c
}

robust earth-abundant electrocatalyst materials are urgently needed for achieving scalable and sustainable hydrogen fuel production by water splitting. ${ }^{13}$ In this context, a variety of nonnoble metal catalysts, such as transition metal compounds including $\mathrm{Mo}, \mathrm{W}, \mathrm{Co}$, Ni, and other metal ions, have been designed as promising candidates for water splitting. ${ }^{\mathbf{1 4 - 2 5}}$ However, in light of sustainable hydrogen production and environmental benignity, it is highly desired to develop efficient and scalable metal-free electrocatalysts. ${ }^{26}$

Heteroatom-doped carbon materials have been widely investigated for energy-conversion/storage due to their large specific surface area, tunable electronic structure, abundance and strong tolerance in acidic/alkaline environments. ${ }^{27-30}$ Nevertheless, solely heteroatom-doped carbon materials showed very poor activity in water splitting. ${ }^{13,31}$ Recently, some advances demonstrated that multi-heteroatom (such as N, S, P, and F) codoped carbon materials offer acceptable electrocatalytic activity for water splitting by tuning the Gibbs free energy of $\mathrm{H}^{*}$ absorption. ${ }^{\mathbf{1 3 , 3 2 - 3 6}} \mathrm{Up}$ to date, most of the developed metal-free carbon electrocatalysts are based on the low dimension carbon materials, such as graphene, carbon nanotubes (CNT), and $\mathrm{C}_{3} \mathrm{~N}_{4}$ composites, etc. ${ }^{\mathbf{1 3 , 3 1 , 3 7 , 3 8}}$ In this regard, synthetic polymers are more suitable for fabrication of multiheteroatom-codoped carbon materials due to their versatile structures and large-scale preparation. As a result, ionic liquid 
polymers (PIL) have been explored as the precursors for the preparation of heteroatom-doped porous carbon, stemming from their high mass yield, designable structure and variable multi-heteroatoms codoping. ${ }^{39-42}$

Herein, we reported a facile strategy to fabricate novel N/S codoped micro-/mesoporous carbon (NSPC) material that was achieved by directly carbonization of bovine serum albumin (BSA) and PIL complex (BSA@PIL). The results demonstrated that the resulting NSPC materials have efficient overall water splitting electrocatalytic activity and excellent supercapacitor performance. Specifically, NSPC pyrolyzed at 1000 degrees (NSPC-1000) presented an overpotential as low as $172 \mathrm{mV} v$ s. RHE (without iR correction) to achieve a current density of 10 $\mathrm{mA} \mathrm{cm}{ }^{-2}$ with a small Tafel slope of $44.3 \mathrm{mV} \mathrm{dec}^{-1}$ in $0.5 \mathrm{M}$ $\mathrm{H}_{2} \mathrm{SO}_{4}$ for HER and a low overpotential of $460 \mathrm{mV} v s$. RHE in 0.1 M KOH for OER. Moreover, beyond applications in water splitting, NSPC-1000 is also a promising candidate for supercapacitor, offering a specific capacitance as high as $495 \mathrm{~F} \mathrm{~g}^{-1}$ at a current density of $0.1 \mathrm{~A} \mathrm{~g}^{-1}$. Such excellent performances of NSPC-1000 in water splitting and supercapacitor originate from the synergistic effects of N/S-codoping and micro-/mesoporous hierarchical architecture.

\section{Experimental}

\section{Synthesis of 1-vinyl-3-cyanomethylimidazolium bromine (CMVImBr)}

1-Vinyl-3-cyanomethylimidazolium bromine (CMVImBr) was synthesized via quaternization of $N$-vinylimidazole with bromoacetonitrile. ${ }^{40}$ In a typical procedure, a $50 \mathrm{~mL}$ flask, equipped with a magnetic stirring bar, was fed with methanol $(2 \mathrm{~mL}), N$ vinylimidazole $(9.411 \mathrm{~g}, 0.1 \mathrm{~mol})$ and bromoacetonitrile (11.995 g, $0.1 \mathrm{~mol})$. The mixture was stirred at ambient temperature to give a white solid. Then, the white solid was dissolved in methanol and the reaction mixture was purified by precipitating three times in ether. Finally, white crystals was obtained and dried at room temperature under vacuum to a constant weight. Yield: $70 \% .{ }^{1} \mathrm{H}$ NMR (hexadeuterodimethyl sulfoxide (DMSO- $\left.d_{6}\right), 400 \mathrm{MHz}, \delta$ ppm, Fig. S1†): 9.58(s, $\left.1 \mathrm{H}\right)$, 8.26(t, 1H), 8.01(t, 1H), 7.36(dd, 1H), 5.99(dd, 1H), 5.63(s, 2H), 5.46(dd, $1 \mathrm{H}) .{ }^{13} \mathrm{C}-\mathrm{NMR}$ (DMSO- $d_{6}, 100 \mathrm{MHz}$, Fig. S2 $\dagger$ ), $\delta(\mathrm{ppm})$ : 137.64, 129.40, 123.92, 120.16, 114.99, 110.21, 37.81 .

\section{Synthesis of poly(1-vinyl-3-cyanomethylimidazolium bromine)} (PIL-Br)

Radical polymerization of CMVImBr was performed in dimethyl sulfoxide using 2,2-azobisisobutyronitrile (AIBN) as an initiator. ${ }^{40}$ In a typical procedure, a $250 \mathrm{~mL}$ round-bottomed flask equipped with a reflux condenser, magnetic stirring bar, and thermometer was charged with $10.0 \mathrm{~g}$ of CMVImBr, $200 \mathrm{mg}$ of AIBN, and $200 \mathrm{~mL}$ of DMSO. The flask was flushed with nitrogen after three freeze-thaw cycles. The mixture was heated at $75{ }^{\circ} \mathrm{C}$ for $24 \mathrm{~h}$. After cooling down, the reaction mixture was purified by precipitating twice from THF. A light yellow solid was obtained and dried at $65{ }^{\circ} \mathrm{C}$ under vacuum to a constant weight.
Yield: $65 \% .{ }^{1} \mathrm{H}-\mathrm{NMR}$ (DMSO- $d_{6}, \delta$ ppm, Fig. S3†): 8.9-9.6 $(1 \mathrm{H})$, 7.0-8.4 (2H), 5.2-5.7 (2H), 3.5-4.6 (1H), 1.9-2.7 (2H).

\section{Preparation of poly(3-cyanomethyl-1-vinyl imidazolium bis(trifluoromethanesulfonyl)imide) (PIL-Tf $\left.{ }_{2} \mathrm{~N}\right)$}

PCMVImTf $_{2} \mathrm{~N}$ was prepared through the anion exchange of PIL$\mathrm{Br}$ in water. ${ }^{41}$ Typically, PIL-Br (3.0 g) was added to $390 \mathrm{~mL}$ of water under gentle heating. After complete dissolution and cooling down, $120 \mathrm{~mL}$ of an aqueous solution containing 1.05 molar equivalents of lithium bis(trifluoromethanesulfonyl) imide salt was added under vigorous stirring. The precipitate was filtered and washed with water several times. The product was dried at $50{ }^{\circ} \mathrm{C}$ to constant weight under high vacuum. Yield: $50 \%$.

\section{Fabrication of the BSA@PIL-Tf ${ }_{2} \mathrm{~N}$ complex and NSPC materials}

BSA@PIL-Tf $\mathrm{T}_{2} \mathrm{~N}$ complex was fabricated according to the reported methods. ${ }^{42}$ PIL-Tf ${ }_{2} \mathrm{~N}$ and BSA $(7: 1, \mathrm{w} / \mathrm{w})$ were fully dissolved in $N, N$-dimethylformamide (DMF) and water mixed solvent $(5: 2, \mathrm{v} / \mathrm{v})$. The as-formed homogeneous solution was then cast onto a pre-cleaned glass slide and dried at $75{ }^{\circ} \mathrm{C}$ for $3 \mathrm{~h}$. Then, the complex was immersed into an ammonia aqueous solution $(0.5 \mathrm{wt} \%)$. The free $\mathrm{COOH}$ groups on BSA were immediately deprotonated by excessive $\mathrm{NH}_{3}$ into carboxylate anions $\left(\mathrm{COO}^{-} \mathrm{NH}_{4}^{+}\right)$, triggering the spontaneous ionic crosslinking between BSA and PIL-Tf ${ }_{2} \mathrm{~N}$. This material is termed as BSA@PIL.

For carbonization, the as-prepared BSA@PIL- $\mathrm{Tf}_{2} \mathrm{~N}$ complex was loaded into an alumina crucible in an oven and carbonized under argon flow. The sample was heated up to $800-1000{ }^{\circ} \mathrm{C}$ $\left(10{ }^{\circ} \mathrm{C} \min ^{-1}\right)$ and kept for $1 \mathrm{~h}$ at this temperature. Then, the furnace was cooled down to room temperature. The samples are defined as NSPC-800, NSPC-900 and NSPC-1000 in accordance with the carbonization temperatures.

\section{Characterizations}

Proton and carbon nuclear magnetic resonance spectroscopy $\left({ }^{1} \mathrm{H}\right.$ and $\left.{ }^{13} \mathrm{C} \mathrm{NMR}\right)$ were recorded using a Brucker AM $400 \mathrm{MHz}$ spectrometer at $25{ }^{\circ} \mathrm{C}$. Gel permeation chromatography (GPC) was conducted at $35^{\circ} \mathrm{C}$ in a Waters-column with DMF as the

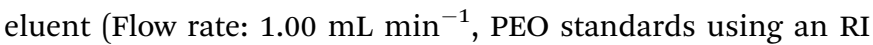
detector). A field emission scanning electron microscope (FESEM, FEI Quanta 600 FEG) was used to acquire SEM images. Transmission electron microscope (TEM), high-resolution TEM (HRTEM) images, selected-area electron diffraction (SAED) patterns, and the HAADF-STEM-EDS data were taken using a Tecnai G20 transmission electron microscope operated at 200 $\mathrm{kV}$. X-ray diffraction (XRD) patterns were collected on a Philips X'Pert Pro diffractometer using $\mathrm{Cu} \mathrm{K}_{\alpha}(\lambda=1.541 \AA)$ radiation source. X-ray photoelectron spectroscopy (XPS) data were collected using a PerkinElmer PHI-5702 multifunctional X-ray photoelectron spectroscope (Physical Electronics, Waltham, Massachusetts) with $\mathrm{Al} \mathrm{K}_{\alpha}$ radiation $(1486.6 \mathrm{eV}$ ) as the excitation source. The adventitious carbon $1 \mathrm{~s}$ peak was calibrated at $285 \mathrm{eV}$ and used as an internal standard to compensate for any 
charging effects. Raman measurements were performed using a Horiba JY-HR800 with the excitation wavelength of $532 \mathrm{~nm}$ and laser power of $100 \mathrm{~mW}$ at room temperature. Elemental analysis (EA) was conducted on Vario EL analyzer (Elementar, Germany). Nitrogen adsorption-desorption isotherm measurements were conducted using a Micromeritics ASAP 2020 volumetric adsorption analyzer at $77 \mathrm{~K}$. The Brunauer-EmmettTeller method was utilized to calculate the surface area of each sample. Pore size distributions were determined from the adsorption branches of the isotherms based on density functional theory.

\section{Electrochemical measurements}

Electrochemical measurements were conducted using a conventional three-electrode electrochemical cell at room temperature on a CHI 660e station (Chenhua, Shanghai, China). A glassy carbon electrode (GCE) with a diameter of $3 \mathrm{~mm}$ was used as the working electrode. The counter electrode and reference electrode were a platinum wire electrode and a saturated calomel electrode (SCE, $0.24 \mathrm{~V}$ vs. a reversible hydrogen electrode (RHE)), respectively. The working electrodes were fabricated similar to the reported method. ${ }^{43}$ A catalyst ink was prepared using one part (by mass) of catalysts and 11 parts of $0.5 \mathrm{wt} \%$ Nafion in ethanol. After sonicating thoroughly, $10 \mu \mathrm{L}$ of the ink was dispensed onto GCE to completely cover the current collector with a thin film of catalyst. The catalyst loading was $0.47 \mathrm{mg} \mathrm{cm}^{-2}$ on the working electrode. Linear sweep voltammetry (LSV) measurements were performed from $0.1 \mathrm{~V}$ to $-0.7 \mathrm{~V}$ with a scan rate of $5 \mathrm{mV} \mathrm{s}^{-1}$ in $0.5 \mathrm{M} \mathrm{H}_{2} \mathrm{SO}_{4}$ solution for HER, $-0.95 \mathrm{~V}$ to $-1.45 \mathrm{~V}$ with a scan rate of $2 \mathrm{mV} \mathrm{s}^{-1}$ in $1.0 \mathrm{M} \mathrm{KOH}$ solution for HER, and $0 \mathrm{~V}$ to $0.8 \mathrm{~V}$ with a scan rate of $2 \mathrm{mV} \mathrm{s}^{-1}$ in $0.1 \mathrm{M} \mathrm{KOH}$ solution for OER. To fabricate CC-supported catalyst, carbon cloth $(0.8 \times 0.5 \mathrm{~cm})$ was utilized as the current collector. Typically, NSPC-1000 (10 mg) and Nafion ethanol dispersion solution ( $1 \mathrm{~mL}, 0.1 \mathrm{wt} \%$ ) were mixed thoroughly by ultrasonicating for $20 \mathrm{~min}$. Then, the uniform suspension was dropped on CC and left to dry at room temperature (this yielded a loading of $0.25 \mathrm{mg} \mathrm{cm} \mathrm{cm}^{-2}$ on CC). All applied potentials, measured against an SCE electrode, are reported versus the reversible hydrogen electrode (RHE) according to $E(v s$. RHE) $=$ $E(v s$. $\mathrm{SCE})+0.24+0.059 \times \mathrm{pH}$ without iR correction. To compare the influence of the component of catalysts to HER and OER activities, the electrocatalytic activities of $\mathrm{Pt} / \mathrm{C}$ and the control were measured under similar conditions, and the corresponding electrocatalytic results are included in the main text. Electrochemical impedance spectroscopy (EIS) was performed over the frequency range from $100 \mathrm{kHz}$ to $0.1 \mathrm{~Hz}$ under the potentiostatic mode at the cell voltage close to the overpotentials of $175 \mathrm{mV}, 309 \mathrm{mV}$, and $417 \mathrm{mV}$ (vs. RHE) corresponding to NSPC-1000, NSPC-900 and NSPC-800, respectively. The amperometric $i-t$ curve and open circuit potential (time curve) were measured with the voltage corresponding to their overpotential. Cyclic voltammetry (CV) was measured between $0-1.0 \mathrm{~V}$ at scan rates of 20,30, 50, 100, 200, 300, and $500 \mathrm{mV} \mathrm{s}^{-1}$ in $0.5 \mathrm{M} \mathrm{H}_{2} \mathrm{SO}_{4}$ solution. The galvanostatic charge/discharge (GCD) property was characterized at the current densities of
$0.1,0.2,0.3,0.5,1,2,3$, and $5 \mathrm{~A} \mathrm{~g}^{-1}$ with a cutoff voltage of $0-$ $0.8 \mathrm{~V}$.

\section{Results and discussion}

The structures of the monomers and polymer were characterized using ${ }^{1} \mathrm{H}$ NMR (Fig. S1-S3 in the ESI $\dagger$ ). The results indicated that PIL-Br was prepared successfully. GPC measurement (Fig. S4†) demonstrated that the apparent number-average molecular weight and polydispersity index of PIL-Br was 1.06 $\times 10^{6} \mathrm{~g} \mathrm{~mol}^{-1}$ and 1.36, respectively. Therefore, the apparent number-average molecular weight of $\mathrm{PIL}-\mathrm{Tf}_{2} \mathrm{~N}$ is calculated to be $1.3 \times 10^{6} \mathrm{~g} \mathrm{~mol}^{-1}$. The following three steps are involved in the synthesis of NSPC: (i) BSA@PIL-Tf ${ }_{2} \mathrm{~N}$ complex was prepared by blending BSA and PIL-Tf ${ }_{2} \mathrm{~N}$ with a ratio of $1: 7(\mathrm{w} / \mathrm{w})$ in $N, N$ dimethyl dimethylformamide (DMF)/water $(5: 2, \mathrm{v} / \mathrm{v})$ mixed solvent. (ii) The as-prepared BSA@PIL-Tf ${ }_{2} \mathrm{~N}$ composite was further treated by immersing into ammonia aqueous $(0.5 \mathrm{wt} \%)$ for $2 \mathrm{~h}$ to form interpolyelectrolyte complex. (iii) Direct pyrolysis of BSA@PIL-Tf ${ }_{2} \mathrm{~N}$ composite at different temperatures under an argon flow yielded NSPCs. These samples are denoted as NSPC800, NSPC-900 and NSPC-1000, labeled with different carbonization temperatures (Scheme 1).

Fig. 1a-c shows the scanning electron microscopy (SEM) images of as-prepared samples. As demonstrated in Fig. 1a and $\mathrm{b}$, spherical or irregular particles were produced when BSA or PIL- $\mathrm{Tf}_{2} \mathrm{~N}$ were pyrolyzed separately at $1000{ }^{\circ} \mathrm{C}$ (the samples are denoted as BSA-1000 and PIL-Tf ${ }_{2} \mathrm{~N}-1000$, respectively). However, the particles with rough surfaces can be observed in NSPC-1000 (Fig. 1c). The elemental compositions of NSPC materials were examined using energy dispersive X-ray (EDX) and elemental analysis (EA). As revealed in Fig. 1d and S5, $\uparrow$ NSPCs mainly consist of $\mathrm{C}, \mathrm{O}, \mathrm{N}$ and $\mathrm{S}$ elements. The populations of both $\mathrm{N}$ and $\mathrm{S}$ decreased with enhancing the carbonization temperature from 800 to $1000{ }^{\circ} \mathrm{C}$ (Table $\mathrm{S} 1 \dagger$ and the inset in Fig. 1d). It should be stated that $3.58 \%$ of $\mathrm{N}$ and $2.01 \%$ of $\mathrm{S}$ still existed in NSPC-1000. These values are comparable to some welldefined N, S-codoped porous carbon. ${ }^{\mathbf{3 4 4 4}}$

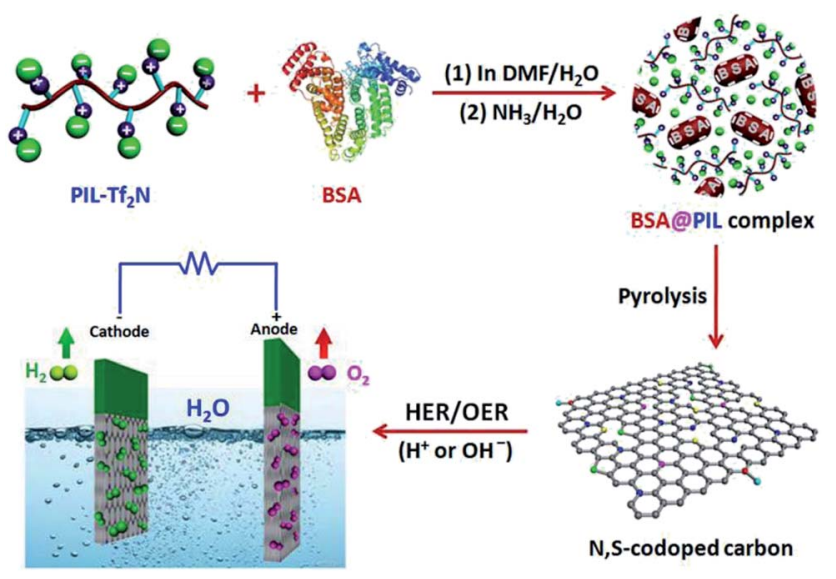

Scheme 1 Schematic synthesis of NSPC and its application in electrocatalytic water splitting via HER and OER. 


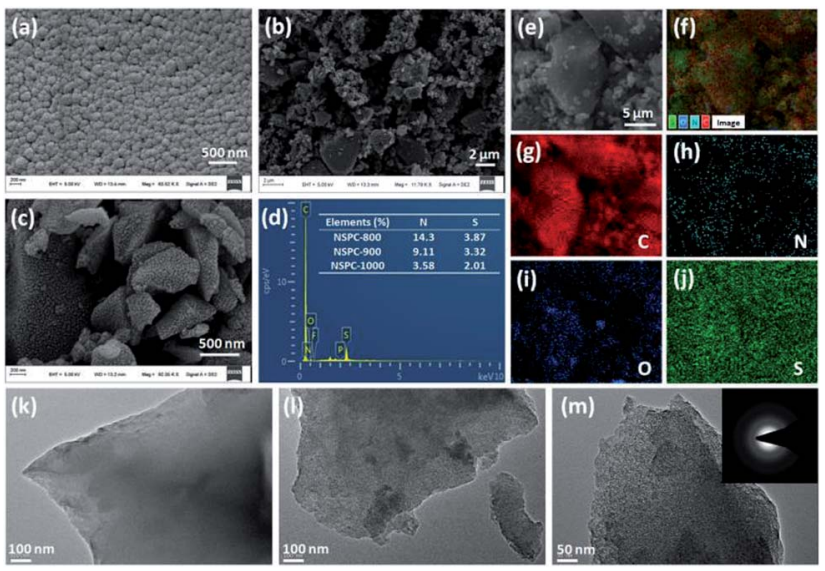

Fig. 1 Morphologies and the elemental compositions of the carbon materials. (a) BSA-1000; (b) PIL-Tf $2 \mathrm{~N}-1000$; (c) NSPC-1000; (d) energy dispersive X-ray (EDX) spectrum of NSPC-1000. Inset table summarizes the corresponding $N$ and $S$ contents in NSPC-800/900/1000 by EA; (e-j) SEM elemental mapping images of $C, N, O$ and $S$ within NSPC-1000; TEM images of (k): NSPC-800; (l): NSPC-900; (m): NSPC1000. Inset SAED pattern indicates the poorly crystallized structure of NSPC-1000.

Energy-filtered scanning electron microscopy mappings for $\mathrm{C}, \mathrm{N}$ and $\mathrm{S}$ (Fig. 1e-j) show a uniform distribution of $\mathrm{N}$ and $\mathrm{S}$ in the carbon matrix, which is expected due to in situ molecular doping of NSPC with $\mathrm{N}$ and S. ${ }^{42}$

Transmission electron microscopy (TEM) images of NSPC materials demonstrate that all of NSPCs are porous structure (Fig. 1k-m), which will be favorable for the internal mass transport, a mandatory requirement for efficient electrocatalysis. ${ }^{45}$ A selected area electron diffraction (SAED) image (inset in Fig. 1m) suggests that NSPC-1000 is poorly crystallized, which can be partially attributed to the high concentration of heteroatoms. Despite the poorly crystallized characteristic of NSPCs, some ordered concentric nanostructures can still be observed (Fig. S6-S8 $\dagger$ ). It can be seen that more nanostructures can be observed in NSPC-1000, indicating progressive graphitization of NSPC at elevated temperatures from 800 to $1000{ }^{\circ} \mathrm{C}$. The synergy between the $\mathrm{N}$ and $\mathrm{S}$ lone pairs and the $\pi$-system of the $\mathrm{C}$ lattice can dramatically modulate the physicochemical properties of porous carbon materials (e.g., oxidative stability and catalytic activity). ${ }^{46}$

In accordance with the EDX and EA results, the X-ray photoelectron spectroscopy (XPS) spectra also confirm that there exist primarily $\mathrm{C}, \mathrm{O}, \mathrm{N}$ and $\mathrm{S}$ elements in the NSPCs (Fig. 2a). As illustrated in Fig. 2b, the fitted XPS peaks for the N1s orbit of NSPCs can be deconvoluted into five different bands at around 398.2, 399.8, 400.5, 401.5, and $404.6 \mathrm{eV}$, corresponding to pyridinic (N1), pyrrolic (N2), graphitic (N3), oxidized pyridinic (N4) and chemisorbed oxidized nitrogen (N5), respectively. These various $\mathrm{N}$ species lead to different chemical/electronic environments of the neighboring carbon atoms and hence different electrocatalytic activities. ${ }^{42}$ The curve fitting and the corresponding normalized results indicate that pyridinic $\mathrm{N}$ gradually turned into graphitic $\mathrm{N}$ with increasing carbonization temperature. Additionally, the XPS S2p spectrum can be deconvoluted into two predominate peaks at $163.7 \mathrm{eV}$ $(-\mathrm{C}-\mathrm{S}-)$ and $164.8 \mathrm{eV}(-\mathrm{C}=\mathrm{S}-)$ (Fig. 2c), suggesting the successful doping of $\mathrm{S}$ atoms into the carbon skeleton in the carbonization process. ${ }^{34}$

X-ray diffraction (XRD) spectra of NSPC materials (Fig. 2d) shows that all of as-prepared samples have two broad peaks located at $2 \theta=25^{\circ}$ and $44^{\circ}$. They are attributed to the graphite (002) and (101) facets of carbon, respectively. ${ }^{47}$ The intensity of the peaks enhanced with increasing carbonization temperature, indicating the improved graphitization degree of NSPC at the elevated temperature. The Raman spectra shows the $\mathrm{D}$ band $\left(1350 \mathrm{~cm}^{-1}\right), \mathrm{G}$ band $\left(1575 \mathrm{~cm}^{-1}\right)$, 2D band $\left(2685 \mathrm{~cm}^{-1}\right), \mathrm{D}+\mathrm{G}$ band $\left(2902 \mathrm{~cm}^{-1}\right)$, and $3 S$ band $\left(3153 \mathrm{~cm}^{-1}\right)$ of NSPC samples (Fig. 2e). The G-band is associated with the E-vibration mode of the $\mathrm{sp}^{2}$ electron structure, corresponding to the aromatic ring structure carbon of the graphite layer, while the D-band is the Avibrational mode of the diamond-like carbon $\mathrm{sp}^{3}$ electronic structure, corresponding to the defective/disordered $\mathrm{sp}^{3}$ hybridized carbon. ${ }^{48}$ As shown in Fig. $2 \mathrm{e}$, the $I_{\mathrm{D}} / I_{\mathrm{G}}$ relative ratio decreases a little as the temperature increases from 800 to $1000{ }^{\circ} \mathrm{C}$, confirming the better graphitic nature of the $\mathrm{N}$, Scodoped carbon skeleton at higher temperature. ${ }^{49}$

Furthermore, the behaviors of nitrogen adsorption-desorption isotherms and the pore size distribution of NSPC materials are shown in Fig. $2 \mathrm{f}$ and g, respectively. It can be seen that all NSPC materials exhibit a type $\mathrm{I}$ adsorption curve and $\mathrm{H}_{2}$-type hysteresis loop, which clearly confirms that NSPCs are hierarchical in nature, traversing the micro- to mesopore range NSPC1000 presents a Brunauer-Emmett-Teller (BET) surface area of $514.4 \mathrm{~m}^{2} \mathrm{~g}^{-1}$ and a total pore volume of $0.469 \mathrm{~cm}^{3} \mathrm{~g}^{-1}$, contributed by a substantial quantity of tiny mesopores around $2.5-8.0 \mathrm{~nm}$ and micropores around $1.5 \mathrm{~nm}$. The meso-/ micropores are probably induced by the leaving small molecules, including $\mathrm{H}_{2} \mathrm{O}, \mathrm{CO}_{2}, \mathrm{NH}_{3}, \mathrm{H}_{2} \mathrm{~S}$, and $\mathrm{CS}_{2}$, during the carbonization and decomposition process of BSA@PIL-Tf ${ }_{2} \mathrm{~N}$ complexes. ${ }^{50}$ This also accounts for the observed pyrolysis temperature-dependent BET surface areas of different samples. Based on the results discussed above, our synthetic protocol enables a facile fabrication of $\mathrm{N}$ - and S-enriched and hierarchical micro-/mesoporous carbon material. It should be noted that our protocol avoids the postsynthetic treatment and simultaneously retains a large surface area in conjunction with abundant dopant species.

To evaluate the electrocatalytic performance of NSPCs for HER, linear sweep voltammetry (LSV) curves were firstly recorded in a conventional three-electrode electrochemical cell in $\mathrm{N}_{2^{-}}$ saturated $0.5 \mathrm{M} \mathrm{H}_{2} \mathrm{SO}_{4}$ solution. The electrocatalysts were cast on a glass carbon electrode (GCE) as the working electrode. As shown in Fig. 3a, NSPC-1000 demonstrates an unprecedentedly low overpotential of $172 \mathrm{mV}$ ( $v s$. RHE, without iR correction) to achieve a current density of $10 \mathrm{~mA} \mathrm{~cm}{ }^{-2}$ and a small Tafel slope of $44.3 \mathrm{mV} \mathrm{dec}^{-1}$ (Fig. 3c), which are much lower than that of NSPC-800 (416 mV and $108.9 \mathrm{mV} \mathrm{dec}^{-1}$ ) and NSPC-900 (309 mV and $65.7 \mathrm{mV} \mathrm{dec}^{-1}$ ). At the same time, a large number of bubbles produced on the NSPC-1000-loaded working electrode (Fig. S9†). As a controlled experiment, PIL-Tf ${ }_{2} \mathrm{~N}-1000$ exhibits 

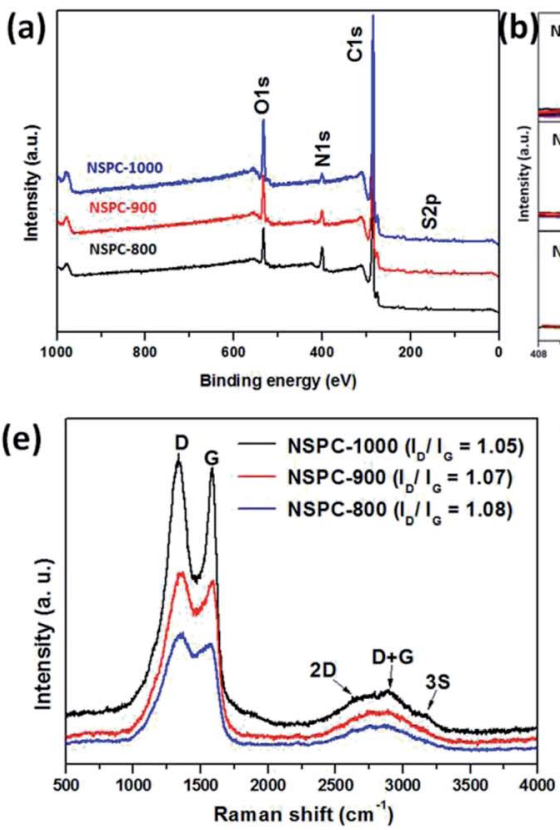
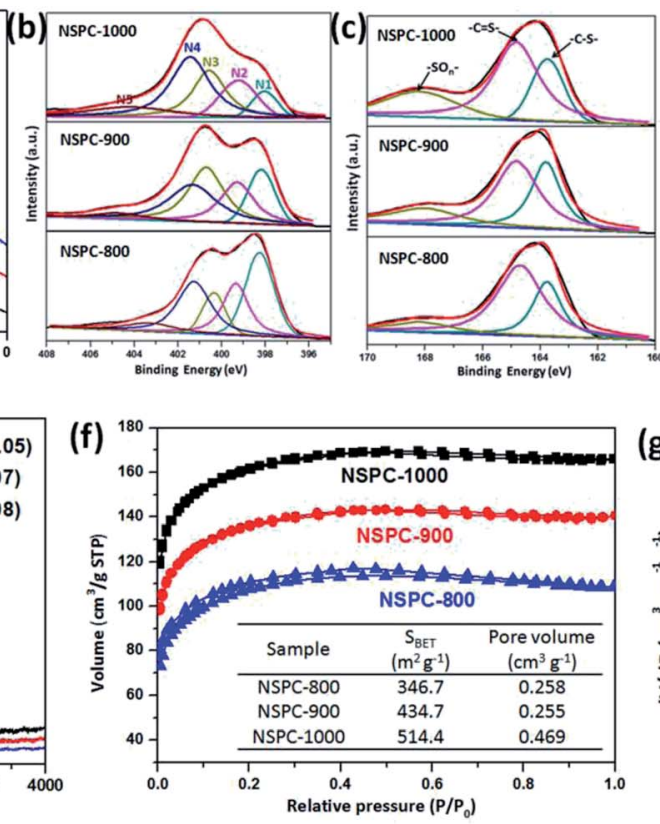
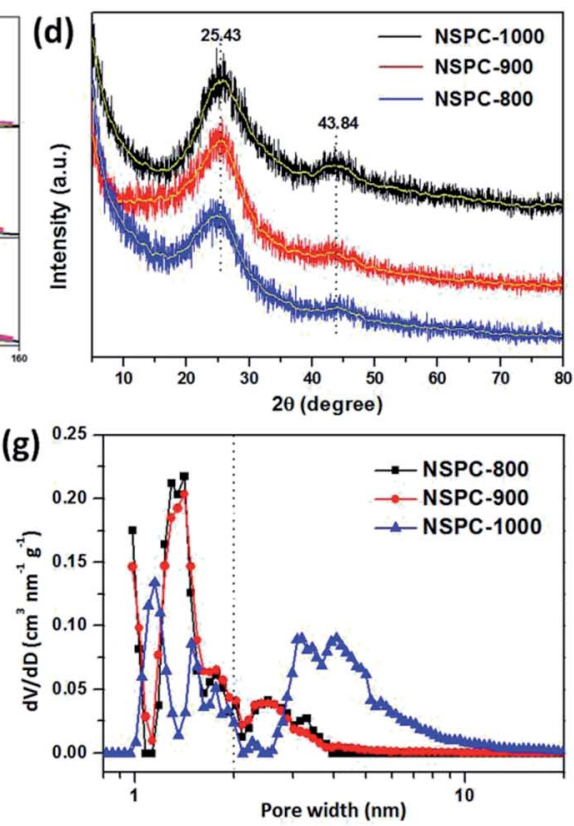

Fig. 2 (a) XPS spectra of NSPC-800/900/1000; fitted XPS peaks for N1s (b) and S2p (c) (N1: pyridinic, N2: pyrrolic, N3: graphitic, N4: oxidized pyridinic, N5: chemisorbed oxidized nitrogen); XRD patterns (d) and Raman spectra (e) of NSPC-800/900/1000; N 2 absorption-desorption isotherms (f) and corresponding pore size distribution (PSD) curves (g) of NSPC-800/900/1000; the inset table presents the data for the specific surface area $\left(S_{B E T}\right)$ and pore volume of NSPC-800/900/1000 based on $\mathrm{N}_{2}$ absorption-desorption isotherm curves.
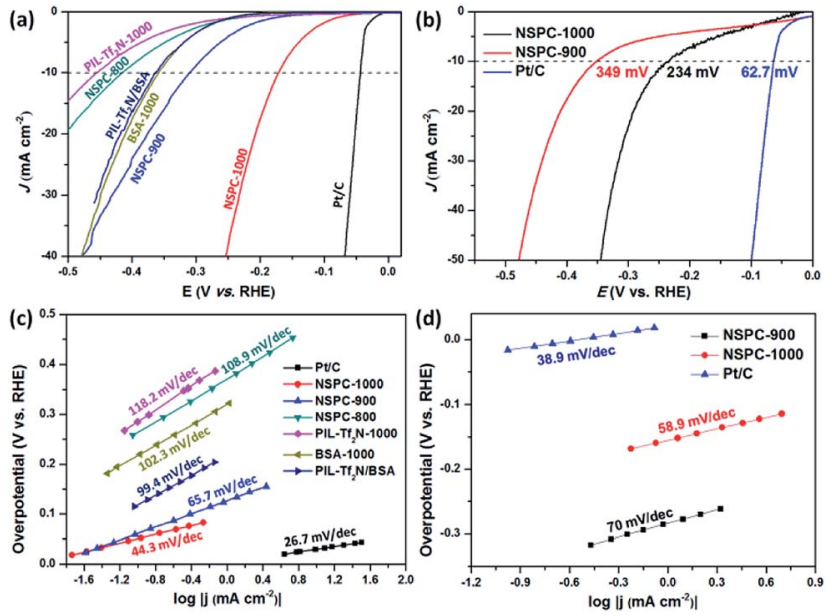

Fig. 3 Electrocatalytic performances of different catalysts in acidic and alkaline conditions. (a) LSV curves of NSPCs in $0.5 \mathrm{M} \mathrm{H}_{2} \mathrm{SO}_{4}$ with a sweep rate of $5 \mathrm{mV} \mathrm{s}^{-1}$, where PIL-Tf 2 N/BSA was prepared by mixing PIL-Tf 2 N-1000 and BSA-1000 (7:1, w/w). (b) LSV curves of NSPC900, NSPC-1000, and Pt/C in $1.0 \mathrm{M} \mathrm{KOH}$ with a sweep rate of $2 \mathrm{mV} \mathrm{s}^{-1}$ (c) and (d) Tafel plots for the data presented in (a) and (b), respectively. All catalysts were loaded on the GCE for HER tests.

the lowest activity among all of these electrocatalysts, while BSA1000 offers the activity closed to NSPC-900. Furthermore, when BSA-1000 was mixed with PIL-Tf ${ }_{2} \mathrm{~N}-1000(1: 7, \mathrm{w} / \mathrm{w})$, the mixture offers a much higher activity than that of $\mathrm{PIL}^{-\mathrm{Tf}_{2} \mathrm{~N}-1000 \text {, and }}$ very close to that of BSA-1000. The results demonstrate that the presence of BSA-1000 play a significant role in achieving higher HER activity.
Generally, HER in acidic electrolyte on the electrode surfaces proceeds via a three-step reaction, which includes a primary discharge step (Volmer reaction, with a Tafel slope of $120 \mathrm{mV}$ $\mathrm{dec}^{-1}$ ) followed by an electrochemical desorption step (Heyrovsky reaction, with a Tafel slope of $40 \mathrm{mV} \mathrm{dec}^{-1}$ ) or a recombination step of adsorbed hydrogen on catalysts (Tafel reaction, with a Tafel slope of $\left.30 \mathrm{mV} \mathrm{dec}^{-1}\right) .{ }^{51-53}$ Typically, a lower Tafel slope predicates a greatly enhanced HER rate at a moderate increase of overpotential. Therefore, the Tafel slope of $44.3 \mathrm{mV}$ $\mathrm{dec}^{-1}$ for NSPC-1000 indicates that the corresponding ratedetermining step most probably involved a combination of both electroreduction of protons into $\mathrm{H}^{*}$ and the subsequent formation of $\mathrm{H}_{2}^{*}$, that is, the Volmer-Tafel reactions. ${ }^{54}$ In addition, $\mathrm{S}$ and $\mathrm{N}$ codoping into $\mathrm{sp}^{2}$ hybridized carbon can produce a unique electronic structures, which will enhance the chemisorptions of intermediate $\mathrm{H}^{*}$ for HER. As a result, a much lower overpotential and smaller Tafel slope could be produced by NSPC-1000.

Furthermore, NSPC materials also exhibit efficient electrocatalytic activity in alkaline conditions. NSPC-1000 and NSPC900 show the overpotential of $234 \mathrm{mV}$ and $349 \mathrm{mV}$ at the current density of $10 \mathrm{~mA} \mathrm{~cm}^{-2}$, respectively (Fig. 3b). Tafel plots shown in Fig. $3 c$ and d show a small Tafel slope of NSPC-1000, demonstrating more favorable kinetics toward HER on the NSPC-1000 electrode. All of these performances presented by NSPC-1000 are comparable to those of many state-of-the-art metal-free and non-noble metal electrocatalysts (Table S2†), as well as some biomass-derived metal-free carbon materials (Table S3†). 
LSV curves were also conducted to evaluate the electrocatalytic activity of NSPC for OER in $0.1 \mathrm{M} \mathrm{KOH}$ solution. As indicated in Fig. 4a, only NSPC-1000 shows efficient electrocatalytic performance under the above conditions. Specifically, NSPC-1000 shows an overpotential of $460 \mathrm{mV}$ at the current density of $10 \mathrm{~mA} \mathrm{~cm}^{-2}$ and the Tafel slope of $88 \mathrm{mV} \mathrm{dec}^{-1}$. This value is comparable to those of some well-defined metal-free electrocatalysts (Table S4 $\dagger$ ). Moreover, continuous HER and OER at a given potential were performed to investigate the durability of the NSPC-1000 electrocatalyst. As shown in Fig. 4b, the current density is stable without an obvious reduction after long-term cycling for over $3.5 \mathrm{~h}$. The robust stability of NSPC1000 provides great potential for sustainable hydrogen and oxygen production in practice.

To gain insight into the electrochemical behavior under HER operating conditions, electrochemical impedance spectroscopy (EIS) measurements were conducted for frequencies from 100 $\mathrm{MHz}$ to $0.01 \mathrm{~Hz}$ at a constant overpotential. From the Nyquist plot, the charge-transfer resistance $\left(R_{\mathrm{ct}}\right)$ and solution resistance $\left(R_{\mathrm{S}}\right)$ can be deduced from the semicircles in the low and high frequency zones, respectively. $R_{\mathrm{S}}$ is used to correct the polarization curves, and $R_{\mathrm{ct}}$ is related to the electrocatalytic kinetics. ${ }^{32,55}$ The result depicted in Fig. S10† reveals a small internal transmission resistance of the NSPCs. The small $R_{\mathrm{ct}}$ enables simple and effective electrical integration that minimized parasitic ohmic losses and enhanced the reaction rate. ${ }^{32}$ Using the $R_{\mathrm{S}}$ obtained from EIS, the iR-corrected polarization curves of the catalysts are shown in Fig. S11. $\dagger$ In the iR-corrected data, NSPC-1000 exhibits a current density of $10 \mathrm{~mA} \mathrm{~cm}^{-2}$ at the lower overpotential of $165 \mathrm{mV}$ in $0.5 \mathrm{M} \mathrm{H}_{2} \mathrm{SO}_{4}$ solution. Therefore, the high catalytic activity of NSPC-1000 resulted from the synergy between its $\mathrm{N}$ and $\mathrm{S}$ codoping, micro/mesoporous and graphitic architecture.
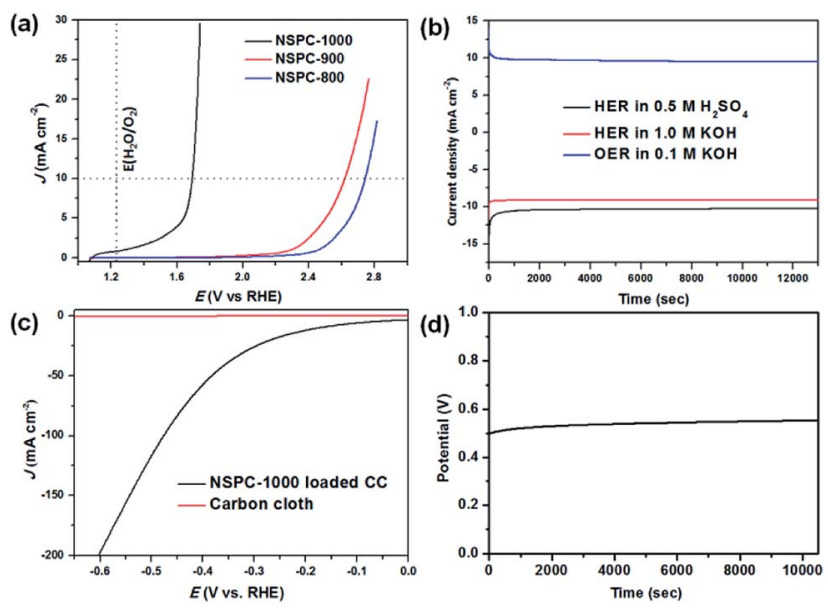

Fig. 4 Electrocatalytic performances of NSPC materials. (a) LSV curves of NSPC-800/900/1000, carbon cloth (CC) and CC-loaded NSPC1000 in $0.1 \mathrm{M} \mathrm{KOH}$ (for OER tests); (b) long-term stability test of NSPC1000 catalyst for HER $\left(-0.45 \mathrm{~V}\right.$ in $0.5 \mathrm{M} \mathrm{H}_{2} \mathrm{SO}_{4},-1.38 \mathrm{~V}$ in $\left.1.0 \mathrm{M} \mathrm{KOH}\right)$ and OER (0.8 V in $0.1 \mathrm{M} \mathrm{KOH})$; (c) LSV curve of water electrolysis using NSPC-1000 as HER and OER catalysts (both loaded into CC at a loading of $0.25 \mathrm{mg} \mathrm{cm}^{-2}$ based on the active mass) in $0.5 \mathrm{M} \mathrm{H}_{2} \mathrm{SO}_{4}$; (d) the stability test of the electrolyzer at $10 \mathrm{~mA} \mathrm{~cm}^{-2}$.
Since NSPC-1000 has been shown to be an efficient and stable bifunctional electrocatalyst for both HER and OER, we loaded NSPC-1000 into a commercially available carbon cloth (CC) and used it as both the anode and the cathode for electrochemical water splitting in $0.5 \mathrm{M} \mathrm{H}_{2} \mathrm{SO}_{4}$ solution. As demonstrated in Fig. 4c, the current density increases gradually with increase of potential, and the current density of $10 \mathrm{~mA}$ $\mathrm{cm}^{-2}$ can be achieved at $175 \mathrm{mV}$, which is very close to that by NSPC-1000 loaded on GCE. As demonstrated in Fig. S12 and Video $\mathrm{S} 1 \uparrow$, a large number of bubbles were produced from both of the electrodes under the experimental potential, indicating that NSPC-1000 is an efficient electrocatalyst for water splitting. The long-term stability of NSPC-1000-loaded CC electrodes was also evaluated, and the duration of the electrolyzer was conducted at $10 \mathrm{~mA} \mathrm{~cm}{ }^{-2}$ in $0.5 \mathrm{M} \mathrm{H}_{2} \mathrm{SO}_{4}$ solution at room temperature. We can see that the cell shows excellent stability at nearly $3 \mathrm{~h}$ (Fig. 4d). Consequently, NSPC materials derived from biomacromolecules and PIL complex, as a novel and versatile metal-free highly efficient electrocatalyst, pave a new pathway for designing water splitting catalysts and show substantial potential for use in renewable energy applications.

In addition to the promising electrocatalytic activity for HER and OER, heteroatom-doped carbonaceous materials have also been demonstrated to be of great potential in supercapacitor. As a proof-of-concept demonstration of the potential application of NSPC in supercapacitor, a symmetric supercapacitor was fabricated using NSPCs as the electrode material. First, cyclic voltammetric (CV) scanning of different NSPCs were conducted in $0.5 \mathrm{M} \mathrm{H}_{2} \mathrm{SO}_{4}$ solution between 0 and $1.0 \mathrm{~V}$, with the obtained curves shown in Fig. S13. $\dagger$ The results show that all of these CV curves exhibit rectangular-like shapes (Fig. S13a and $\mathrm{b} \dagger$ ), indicating the ideal capacitive behavior and good charge propagation within the electrodes. ${ }^{56}$ Under identical conditions, the NSPC-1000 curve provides the largest encircled area among these electrode materials, suggesting that NSPC-1000 shows better capacitance behavior. The CV curves measured with scan rates ranging from 20 to $500 \mathrm{mV} \mathrm{s}^{-1}$ (Fig. S13c†) also exhibit a rectangular-like shape, and the encircled area shrinks simultaneously. Additionally, when the potential window is negatively shifted and still kept within $1.0 \mathrm{~V}$, the curves become increasingly sickle-like, similar to the behavior of the metal catalysts (Fig. S13d $\dagger$ ). ${ }^{57}$ Meanwhile, the working electrode loaded with NSPC-1000 began to bubble during the test, and the bubbling is accelerated with the negative voltage shifting. This phenomenon also demonstrates that NSPC-1000 is an excellent electrocatalyst for water splitting.

Then, galvanostatic charge/discharge (GCD) measurements were conducted to evaluate the capacitance performance of the above materials at $0.1 \mathrm{~A} \mathrm{~g}^{-1}$ in a stable voltage window between 0 and $0.8 \mathrm{~V}$. As illustrated in Fig. $5 \mathrm{a}$ and b, the linear voltagetime profiles and the triangular shape of the charge/discharge plots without an obvious iR drop illustrate the small mass transfer resistance and good charge propagation behavior in the electrode materials. The specific capacitance $\left(C_{\mathrm{s}}\right)$ can be calculated from the discharge curves according to the following equation: ${ }^{58}$ 

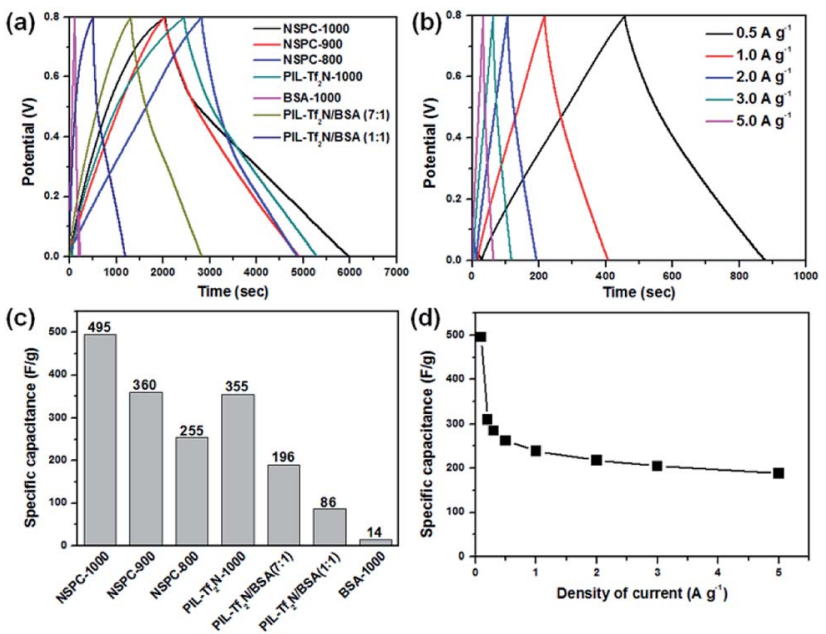

Fig. 5 Electrochemical performances of carbon materials for supercapacitor. (a) Galvanostatic charge/discharge (GCD) curves of different carbon materials at a current density of $0.1 \mathrm{~A} \mathrm{~g}^{-1}$. (b) GCD curves of NSPC-1000 electrode material at different current densities. (c) Discharge capacitances of different carbon materials corresponding to the data in (a). (d) Discharge capacitances at various current densities corresponding to the data in (b).

$$
C_{\mathrm{s}}=C / m=(I \Delta t) /(\Delta V m)
$$

where $C_{\mathrm{s}}$ is the specific capacitance $\left(\mathrm{F} \mathrm{g}^{-1}\right), I$ is the discharge current (A), $\Delta t$ is the discharging time (s), $\Delta V$ is the potential range during the discharge process $(\mathrm{V})$, and $m$ is the mass of the active material within the electrode $(\mathrm{g})$. Remarkably, the $C_{\mathrm{s}}$ value of $495 \mathrm{~F} \mathrm{~g}^{-1}$ is found for NSPC-1000, which is much higher than those of the other carbonaceous materials. As depicted in Fig. $5 \mathrm{c}$, we can infer that the presence of PIL-Tf ${ }_{2} \mathrm{~N}$ and high pyrolysis temperature are beneficial for the enhancement of the capacitance of the carbon material, while the presence of BSA1000 is disadvantageous for the capacitance. Some previous studies have demonstrated that the doping of $\mathrm{S}$ into $\mathrm{sp}^{2}$ hybridized carbon can produce a unique electronic structure and benefit to improve the capacitance performance. ${ }^{59-61}$ Thus, the higher specific capacitance of NSPCs was probably because that PIL-Tf ${ }_{2} \mathrm{~N}$ could contributes more sulfur element content to the hybrid carbon material (Table S1 $\dagger$ ). Fig. $5 \mathrm{~d}$ reveals that $C_{\mathrm{s}}$ of NSPC-1000 decreases rapidly with enhanced current density. However, $C_{\mathrm{s}}$ of $194 \mathrm{~F} \mathrm{~g}^{-1}$ can still be retained at a current density as high as $5 \mathrm{~A} \mathrm{~g}^{-1}$. The above results demonstrate that $\mathrm{N}$, Scodoped porous carbon produced at higher temperature can show better capacitance performance.

\section{Conclusions}

In summary, a facile protocol for fabrication of $\mathrm{N}$, S-codoped porous carbon was developed via carbonization of the BSA@PIL-Tf ${ }_{2} \mathrm{~N}$ complex. Our studies reveal that the novel N, Scodoped carbons featured micro-/mesoporous architectures. Due to their large surface area and good electron/electrolyte transport properties, NSPC materials are demonstrated to be effective bifunctional HER/OER electrocatalysts with excellent activity and stability. In particular, NSPC-1000 affords the lower overpotential and smaller Tafel slope, outperforming many well-defined non-precious metals and metal-free electrocatalysts. Simultaneously, the newly developed NSPC materials also exhibit outstanding capacitance performance. In view of the low cost of biomacromolecules and the facile synthesis of PIL, NSPC material is a promising alternative to (non)noble metal-based catalysts for water splitting, as well as for supercapacitor and other electrochemistry applications. Our facile protocol via the combination of biomacromolecules and synthetic polymers opens up a new avenue for devising effective, readily scalable and metal-free $\mathrm{N}$, S-codoped carbon electrode materials for multi-functional applications.

\section{Conflicts of interest}

There are no conflicts to declare.

\section{Acknowledgements}

The authors are grateful for the financial support given by the National Natural Science Foundation of China (Project No. 21774101, 21474080).

\section{Notes and references}

1 M. I. Hoffert, K. Caldeira, G. Benford, D. R. Criswell, C. Green, H. Herzog, A. K. Jain, H. S. Kheshgi, K. S. Lackner, J. S. Lewis, H. D. Lightfoot, W. Manheimer, J. C. Mankins, M. E. Mauel, L. J. Perkins, M. E. Schlesinger, T. Volk and T. M. L. Wigley, Science, 2002, 298, 981-987.

2 Y. Zhou, M. Hejazi, S. Smith, J. Edmonds, H. Li, L. Clarke, K. Calvina and A. Thomsona, Energy Environ. Sci., 2015, 8, 2622-2633.

3 M. W. Kanan and D. G. Nocera, Science, 2008, 321, 10721075.

4 Y. Liang, Y. Li, H. Wang, J. Zhou, J. Wang, T. Regier and H. Dai, Nat. Mater., 2011, 10, 780-786.

5 S. Dunn, Int. J. Hydrogen Energy, 2002, 27, 235-264.

6 M. S. Dresselhaus and I. L. Thomas, Nature, 2001, 414, 332337.

7 J. A. Turner, Science, 2004, 305, 972-974.

8 R. Subbaraman, D. Tripkovic, D. Strmcnik, K. C. Chang, M. Uchimura, A. P. Paulikas, V. Stamenkovic and N. M. Markovic, Science, 2011, 334, 1256-1260.

9 N. C. Cheng, N. B. Banis, J. Liu, A. Riese, X. Li, R. Y. Li, S. Y. Ye, S. N. Knights and X. L. Sun, Adv. Mater., 2015, 27, 277-281.

10 H. F. Lv, Z. Xi, Z. Z. Chen, S. J. Guo, Y. S. Yu, W. L. Zhu, Q. Li, X. Zhang, M. Pan, G. Lu, S. C. Mu and S. H. Sun, J. Am. Chem. Soc., 2015, 137, 5859-5862.

11 P. Xiao, W. Chen and X. Wang, Adv. Energy Mater., 2015, 5, 1500985.

12 Y. Lee, J. Suntivich, K. J. May, E. E. Perry and Y. Shao-Horn, J. Phys. Chem. Lett., 2012, 3, 399-404.

13 L. Dai, Y. Xue, L. Qu, H. J. Choi and J. B. Baek, Chem. Rev., 2015, 115, 4823-4892. 
14 Z. Lu, H. Wang, D. Kong, K. Yan, P. C. Hsu, G. Zheng, H. Yao, Z. Liang, X. Sun and Y. Cui, Nat. Commun., 2014, 5, 4345.

15 W. F. Chen, J. T. Muckerman and E. Fujita, Chem. Commun., 2013, 49, 8896-8909.

16 L. A. Stern, L. Feng, F. Song and X. Hu, Energy Environ. Sci., 2015, 8, 2347-2351.

17 J. Wang, W. Cui, Q. Liu, Z. Xing, A. M. Asiri and X. Sun, Adv. Mater., 2016, 28, 215-230.

18 D. Voiry, H. Yamaguchi, J. Li, R. Silva, D. C. B. Alves, T. Fujita, M. Chen, T. Asefa, V. B. Shenoy, G. Eda and M. Chhowalla, Nat. Mater., 2013, 12, 850-855.

19 M. R. Gao, J. X. Liang, Y. R. Zheng, Y. F. Xu, J. Jiang, Q. Gao, J. Li and S. H. Yu, Nat. Commun., 2015, 6, 5982.

20 Y. Liang, H. Wang, J. Zhou, Y. Li, J. Wang, T. Regier and H. Dai, J. Am. Chem. Soc., 2012, 134, 3517-3523.

21 H. Vrubel and X. L. Hu, Angew. Chem., Int. Ed., 2012, 51, 12703-12706.

22 X. Zou and Y. Zhang, Chem. Soc. Rev., 2015, 44, 5148-5180.

23 Y. Yang, H. L. Fei, G. D. Ruan, C. S. Xiang and J. M. Tour, Adv. Mater., 2014, 26, 8163-8168.

24 Y. Zhao, K. Kamiya, K. Hashimoto and S. Nakanishi, Angew. Chem., Int. Ed., 2013, 52, 13638-13641.

25 D. Y. Wang, M. Gong, H. L. Chou, C. J. Pan, H. A. Chen, Y. P. Wu, M. C. Lin, M. Y. Guan, J. Yang, C. W. Chen, Y. L. Wang, B. J. Hwang, C. C. Chen and H. J. Dai, J. Am. Chem. Soc., 2015, 137, 1587-1592.

26 Z. L. Wang, X. F. Hao, Z. Jiang, X. P. Sun, D. Xu, J. Wang, H. X. Zhong, F. L. Meng and X. B. Zhang, J. Am. Chem. Soc., 2015, 137, 15070-15073.

27 Z. W. Liu, F. Wang, M. Li and Z. H. Ni, RSC Adv., 2016, 6, 37500-37505.

28 Z. Xiao, X. Y. Gao, M. H. Shi, G. Y. Ren, G. Z. Xiao, Y. Zhu and L. Jiang, RSC Adv., 2016, 6, 86401-86409.

29 J. Deng, M. M. Li and Y. Wang, Green Chem., 2016, 18, 48244854 .

30 S. J. Yuan and X. H. Dai, RSC Adv., 2015, 5, 45827-45835.

31 Y. Zheng, Y. Jiao, Y. Zhu, L. H. Li, Y. Han, Y. Chen, A. Du, M. Jaroniec and S. Z. Qiao, Nat. Commun., 2014, 5, 3783.

32 J. Duan, S. Chen, M. Jaroniec and S. Z. Qiao, ACS Nano, 2015, 9, 931-940.

33 Y. Zheng, Y. Jiao, L. H. Li, T. Xing, Y. Chen, M. Jaroniec and S. Z. Qiao, ACS Nano, 2014, 8, 5290-5296.

34 J. Zhang, Z. Zhao, Z. Xia and L. Dai, Nat. Nanotechnol., 2015, 10, 444-452.

35 J. Zhang, L. Qu, G. Shi, J. Liu, J. Chen and L. Dai, Angew. Chem., Int. Ed., 2016, 128, 2270-2274.

36 C. Hu and L. Dai, Adv. Mater., 2017, 29, 1604942.

37 M. Zhang and L. Dai, Nano Energy, 2012, 1, 514-517.

38 K. Qu, Y. Zheng, Y. Jiao, X. Zhang, S. Dai and S. Z. Qiao, Adv. Energy Mater., 2017, 7, 1602068.

39 J. Yuan, D. Mecerreyes and M. Antonietti, Prog. Polym. Sci., 2013, 38, 1009-1036.
40 J. Yuan, C. Giordano and M. Antonietti, Chem. Mater., 2010, 22, 5003-5012.

41 J. Yuan, A. G. Márquez, J. Reinacher, C. Giordano, J. Janek and M. Antonietti, Polym. Chem., 2011, 2, 1654-1657.

42 H. Wang, S. Min, C. Ma, Z. Liu, W. Zhang, Q. Wang, D. Li, Y. Li, S. Turner, Y. Han, H. Zhu, E. Abou-hamad, M. N. Hedhili, J. Pan, W. Yu, K. W. Huang, L. J. Li, J. Yuan, M. Antonietti and T. Wu, Nat. Commun., 2017, 8, 13592.

43 M. Lei, P. G. Li, L. H. Li and W. H. Tang, J. Power Sources, 2011, 196, 3548-3552.

44 Z. Pei, H. Li, Y. Huang, Q. Xue, Y. Huang, M. Zhu, Z. Wang and C. Zhi, Energy Environ. Sci., 2017, 10, 742-749.

45 J. C. Lytle, J. M. Wallace, M. B. Sassin, A. J. Barrow, J. W. Long, J. L. Dysart, C. H. Renninger, M. P. Saunders, N. L. Brandell and D. R. Rolison, Energy Environ. Sci., 2011, 4, 1913-1925.

46 K. Gong, F. Du, Z. Xia, M. Durstock and L. Dai, Science, 2009, 323, 760-764.

47 F. Su, C. K. Poh, J. S. Chen, G. Xu, D. Wang, Q. Li, J. Lin and X. W. Lou, Energy Environ. Sci., 2011, 4, 717-724.

48 J. Liang, Y. Jiao, M. Jaroniec and S.-Z. Qiao, Angew. Chem., Int. Ed., 2012, 51, 11496-11500.

49 J. Duan, Y. Zheng, S. Chen, Y. Tang, M. Jaroniec and S.-Z. Qiao, Chem. Commun., 2013, 49, 7705-7707.

50 J. Liang, Y. Zheng, J. Chen, J. Liu, D. Hulicova-Jurcakova, M. Jaroniec and S. Z. Qiao, Angew. Chem., Int. Ed., 2012, 51, 3892-3896.

51 J. G. N. Thomas, Trans. Faraday Soc., 1961, 57, 1603-1611.

52 Y. Li, H. Wang, L. Xie, Y. Liang, G. Hong and H. Dai, J. Am. Chem. Soc., 2011, 133, 7296-7299.

53 Z. Chen, D. Cummins, B. N. Reinecke, E. Clark, M. K. Sunkara and T. F. Jaramillo, Nano Lett., 2011, 11, 4168-4175.

54 X. Liu, W. Zhou, L. Yang, L. Li, Z. Zhang, Y. Ke and S. Chen, J. Mater. Chem. A, 2015, 3, 8840-8846.

55 S. Mao, Z. Wen, S. Ci, X. Guo, K. Ostrikov and J. Chen, Small, 2015, 11, 414-419.

56 P. Simon and Y. Gogotsi, Nat. Mater., 2008, 7, 845-854.

57 C. C. Hu and T. W. Tsou, Electrochem. Commun., 2002, 4, 105-109.

58 Y. Zhu, S. Murali, M. D. Stoller, K. J. Ganesh, W. Cai, P. J. Ferreira, A. Pirkle, R. M. Wallace, K. A. Cychosz, M. Thommes, D. Su, E. A. Stach and R. S. Ruoff, Science, 2011, 332, 1537-1541.

59 X. A. Chen, X. H. Chen, X. Xu, Z. Yang, Z. Liu, L. J. Zhang, X. Q. Xu, Y. Chen and S. M. Huang, Nanoscale, 2014, 6, 13740-13747.

60 L. Sun, J. Liu, Z. Liu, T. Wang, H. Wang and Y. Li, RSC Adv., 2018, 8, 19964-19970.

61 S. Liu, Y. Cai, X. Zhao, Y. Liang, M. Zheng, H. Hu, H. Dong, S. Jiang, Y. Liu and Y. Xiao, J. Power Sources, 2017, 31, 373382. 